\title{
AP-VAS 2012 case report: a case of myeloperoxidase antineutrophil cytoplasmic antibody-positive microscopic polyangiitis with rapidly progressive glomerulonephritis and hearing loss
}

\author{
Maki Tsukamoto - Seiichiro Shimizu - Megumi Koizumi • \\ Nobuo Kitahara • Yoshihiro Ohtaki • Shigeyuki Aoki • \\ Hiroshi Miyakawa
}

Received: 14 August 2012/Accepted: 7 November 2012/Published online: 30 January 2013

(C) Japanese Society of Nephrology 2012

\begin{abstract}
A 74-year-old Japanese woman was admitted to our hospital because of fever, fatigue, and hearing loss associated with vertigo. She had a 1-year history of hearing impairment that got worse gradually and had been treated as otitis media with effusion, but without remarkable improvement. After admission, she developed renal dysfunction associated with hematuria and proteinuria. Laboratory tests showed leukocytosis and elevated C-reactive protein. Myeloperoxidase antineutrophil cytoplasmic antibody (MPO-ANCA) was elevated, but proteinase-3 antineutrophil cytoplasmic antibody (PR3-ANCA) was negative. Renal biopsy revealed pauci-immune focal necrotizing glomerulonephritis with crescents. She was diagnosed as having MPO-ANCA-associated polyangiitis. After treatment with $500 \mathrm{mg}$ methylprednisolone applied intravenously for 3 days, followed by $40 \mathrm{mg}$ prednisolone
\end{abstract}

M. Tsukamoto ( $\square)$

Department of Nephrology and Endocrinology, Graduate School of Medicine, The University of Tokyo, 7-3-1 Hongo, Bunkyo-

ku, Tokyo 1138655, Japan

e-mail: mtsukamoto-tky@umin.ac.jp

\section{S. Shimizu}

Department of Pathology,

Showa General Hospital, Tokyo, Japan

M. Koizumi · N. Kitahara

Department of Otolaryngology,

Showa General Hospital, Tokyo, Japan

Y. Ohtaki $\cdot$ S. Aoki

Department of Respirology,

Showa General Hospital, Tokyo, Japan

H. Miyakawa

Department of Nephrology,

Showa General Hospital, Tokyo, Japan administered orally, renal function recovered completely. Her hearing also improved. Although otolaryngological symptoms are common in PR3-ANCA associated vasculitis, hearing loss is a rare manifestation of MPO-ANCA associated vasculitis (MPO-AAV). Our case suggests that AAV should be considered in the differential diagnosis of hearing loss.

Keywords Myeloperoxidase antineutrophil cytoplasmic antibody - Microscopic polyangiitis - Rapidly progressive glomerulonephritis $\cdot$ Hearing loss

\section{Introduction}

MPO-ANCA-positive microscopic polyangiitis (MPA) is a necrotizing small-vessel vasculitis that commonly manifests as a rapid progressive glomerular nephritis (RPGN), with necrotizing glomerular tufts, alveolar hemorrhage, or interstitial pneumonia [1-3]. Most patients with ANCAassociated glomerulonephritis present with a rapid loss of renal function associated with hematuria, proteinuria, and hypertension [4]. Granulomatosis with polyangiitis (GPA), previously known as Wegener's granulomatosis, is common with ENT symptoms, but for MPA it is rare. This report describes a case of MPO-ANCA-positive MPA with hearing loss followed by renal dysfunction.

\section{Case report}

A 74-year-old woman was admitted to our hospital because of fever, fatigue, and hearing loss associated with vertigo. Her hearing impairment for the prior year, which had been diagnosed as otitis media with effusion, was resistant 
against ventilation tube insertion and clarithromycin. T2weighted MR image showed high signal representing effusion in mastoid cells, which was compatible with otitis media with effusion (Fig. 1).

She had a medical history of acute appendicitis and cataract. Physical examination revealed bilateral hearing loss and coarse crackles on the left side of her lung. No

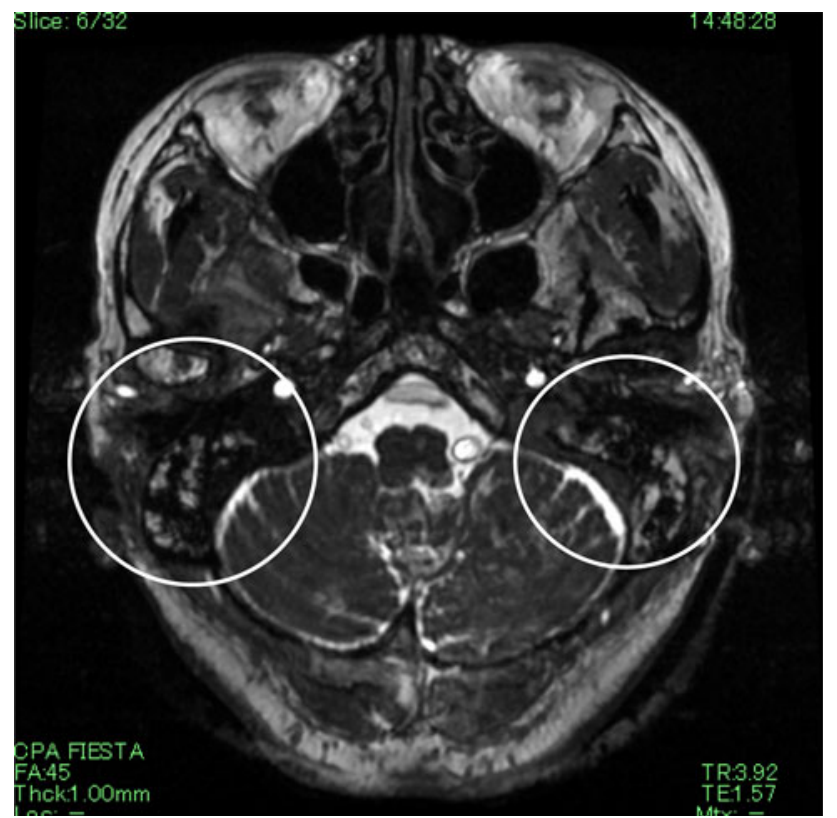

Fig. 1 T2-weighted MR image showed high signal representing effusion in mastoid cells (white circles)

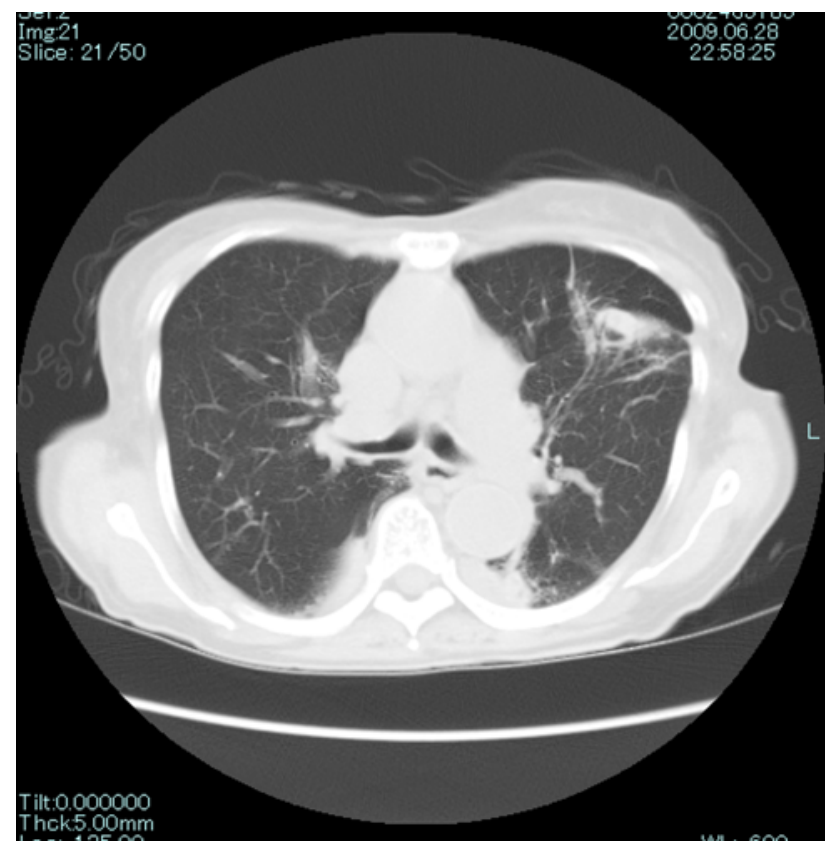

Fig. 2 Plain chest CT showing consolidation in her left lung skin lesion, saddle nose, peripheral neuropathy or arthralgia was found.

Laboratory tests on admission showed leukocytosis $(18,000 / \mu \mathrm{L})$, elevated C-reactive protein $(23.2 \mathrm{mg} / \mathrm{dL})$, positive for MPO-ANCA (100 EU/mL), but negative for PR3-ANCA. Her creatinine was normal $(0.9 \mathrm{mg} / \mathrm{dL})$. No viral or bacterial infection, including tuberculosis, was detected by appropriate tests. Plain CT showed consolidation on her left lung, but normal-sized kidney (Figs. 2, 3). Lung biopsy was performed, but abnormal findings were not obtained.

On day 16 after admission, serum creatinine increased rapidly from 0.9 to $4.5 \mathrm{mg} / \mathrm{dL}$ associated with proteinuria (1.8 g/day) and microscopic hematuria (>100/HPF). Renal biopsy revealed pauci-immune focal necrotizing glomerulonephritis with crescents (Fig. 4). She was diagnosed as having MPO-ANCA positive microscopic polyangiitis and

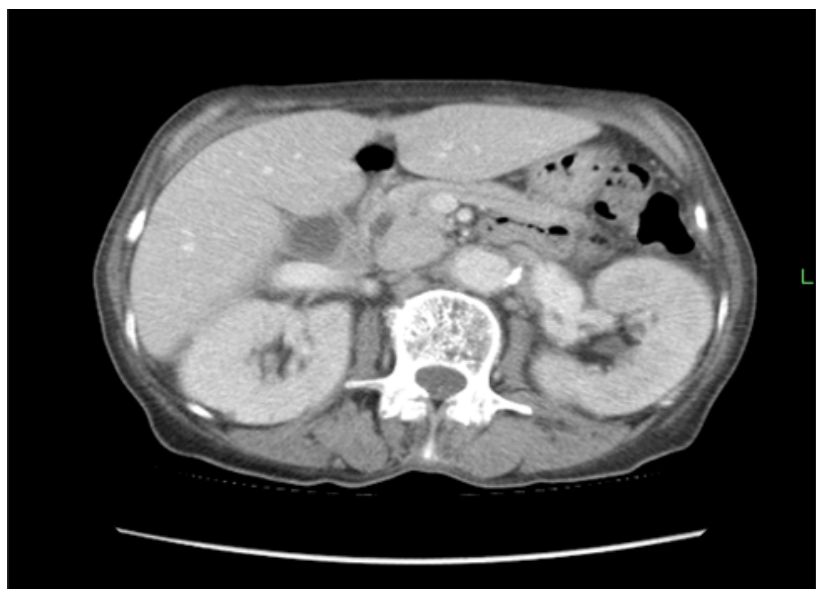

Fig. 3 Plain pelvic CT showing a normal sized kidney

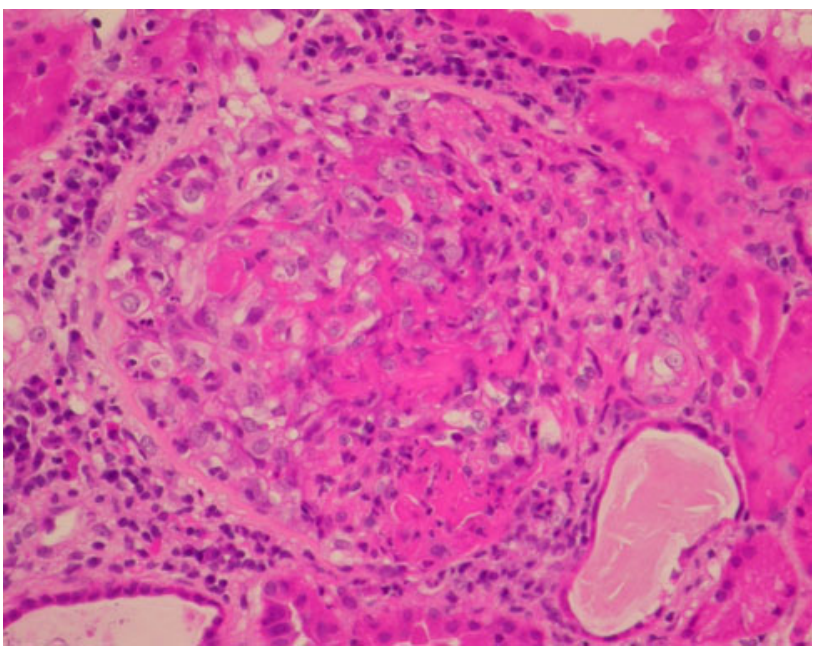

Fig. 4 Renal biopsy image showing focal necrotizing glomerulonephritis with crescents 


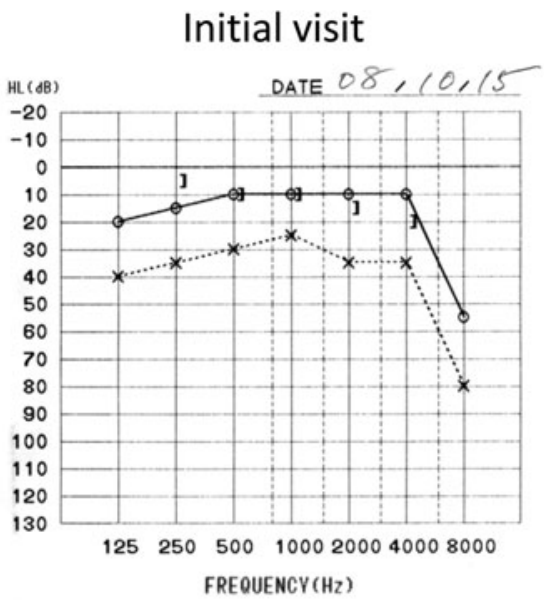

\section{Exacerbation}

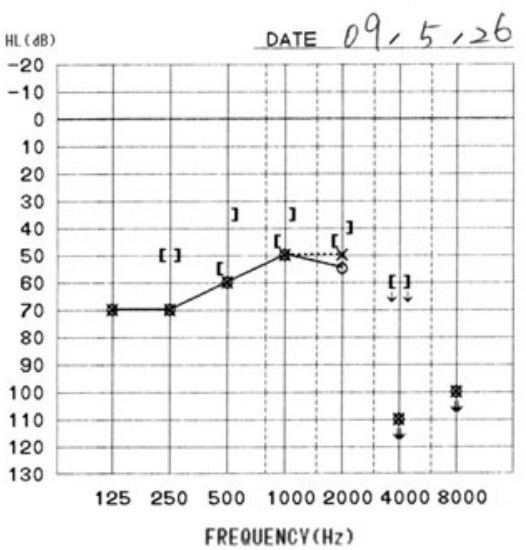

After treatment

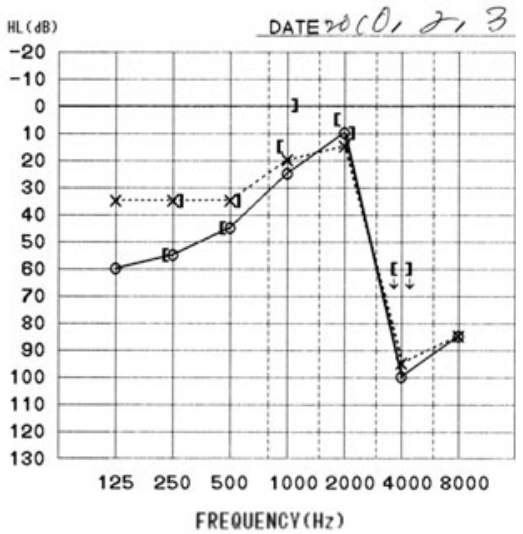

Fig. 5 Audiogram from initial visit to after treatment. It shows improvement after treatment with steroids

was subsequently treated with $500 \mathrm{mg}$ methylprednisolone intravenously for 3 days followed by $40 \mathrm{mg} /$ day prednisolone (oral). After treatment, renal function completely recovered, with disappearance of proteinuria and hematuria. Additionally, an audiogram confirmed that her hearing had improved (Fig. 5).

After discharge, she maintained a stable clinical course and normal renal function. Her hearing also has not worsened.

\section{Discussion}

AAV is characterized by the occurrence of systemic necrotizing vasculitis with multiple organ dysfunction, associated with the presence of ANCA in serum, which is highly specific for the diagnosis of vasculitis (98.6 \%) [5]. GPA, previously known as Wegener's granulomatosis, is a systemic necrotizing granulomatous AAV that mainly affects the upper and lower respiratory tracts and kidneys, whereas MPA is a systemic necrotizing AAV that typically causes RPGN and hemorrhagic pneumonitis. In Japan, ENT symptoms are rare $(15 \%)$ compared with those reported in the UK (64 \%) [6]. In Japan, MPA is regarded as predominant, but GPA is more common in the UK among patients with AAV.

PR3-ANCA is more specific for GPA and MPO-ANCA is more specific for MPA. However, MPO-ANCA-positive GPA and PR3-ANCA-positive MPA are frequently encountered. A previous study showed that $10 \%$ of GPA patients were positive for MPO-ANCA, while $30 \%$ of MPA patients were positive for PR3-ANCA [7]. Our case was positive for only MPO-ANCA and associated with rare manifestation as hearing impairment in Japan. We concluded our case with MPO-ANCA-positive MPA because of a lack of clinical manifestation, suggesting GPA and no detection of granulomatosis in lung and renal biopsy.

Our patient had suffered refractory otitis media with effusion for 1 year. Fortunately, her hearing ability improved together with treatment for MPO-ANCA-positive MPA, but it did not completely recover. Takagi et al. [8] reported their six MPO-ANCA-positive cases with bilateral progressive hearing loss. In their report, initiation of treatment within 2 months improved the outcome of hearing ability, and was able to achieve complete recovery. Our patient achieved only partial recovery. The initiation of treatment occurred more than 2 months after hearing loss developed.

In conclusion, it is sometimes difficult to diagnose AAV with only hearing impairment without systemic symptoms because that symptom associated with AAV is still rare in Japan. However, based on information from our case and another literature review [8-10], early diagnosis and initiation of treatment for AAV can save hearing loss. AAV should be considered in the differential diagnosis of hearing loss that is resistant to conventional treatment.

Conflict of interest None declared.

\section{References}

1. Falk RJ, Jennette JC. Anti-neutrophil cytoplasmic autoantibodies with specificity for myeloperoxidase in patients with systemic vasculitis and idiopathic necrotizing and crescentic glomerulonephritis. N Engl J Med. 1988;318:1651-7.

2. Bosch X, Mirapeix E, Font J, Cervera R, Ingelmo M, Khamashta MA, et al. Anti-myeloperoxidase autoantibodies in patients with necrotizing glomerular and alveolar capillaritis. Am J Kidney Dis. 1992;20:231-9.

3. Geffriaud-Ricouard C, Noel LH, Chauveau D, Houhou S, Grunfeld JP, Lesavre P. Clinical spectrum associated with ANCA 
of defined antigen specificities in 98 selected patients. Clin Nephrol. 1993;39:125-36.

4. Falk RJ, Hogan S, Carey TS, Jennette JC. The glomerular disease collaborative network. Clinical course of anti-neutrophil cytoplasmic autoantibody-associated glomerulonephritis and systemic vasculitis. Ann Intern Med. 1990;113:656-63.

5. Choi HK, Liu S, Merkel PA, Colditz GA, Niles JL. Diagnostic performance of antineutrophil cytoplasmic antibody tests for idiopathic vasculitides: meta analysis with a focus on antimyeloperoxidase antibodies. J Rheumatol. 2001;28:1584-90.

6. Fujimoto S, Watts RA, Kobayashi S, Suzuki K, Ingelmo M, Khamashta MA, et al. Comparison of the epidemiology of antineutrophil cytoplasmic antibody-associated vasculitis between Japan and the UK. Rheumatology (Oxford). 2011;50:1916-20.
7. Lane SE, Watts RA, Shepstone L, Scott DG. Primary systemic vasculitis: clinical features and mortality. QJM. 2005;98:97-111.

8. Takagi D, Nakamura Y, Maguchi S, Furuta Y, Fukuda S. Clinical features of bilateral progressive hearing loss associated with myeloperoxidase-antineutrophil cytoplasmic antibody. Ann Otol Rhinol Laryngol. 2004;113:388-93.

9. Maguchi S, Fukuda S, Chida E, Terayama Y. Myeloperoxidaseantineutrophil cytoplasmic antibody-associated sensorineural hearing loss. Auris Nasus Larynx. 2001;28:S103-6.

10. Okura T, Miyoshi K, Jotoku M, Enomoto D, Irita J, Nagao T, et al. A patient with myeloperoxidase antineutrophil cytoplasmic antibody-positive polyangiitis who developed sensorineural hearing loss and scleritis. Intern Med. 2011;50:1725-8. 